\title{
Alterações da comunicação em pacientes institucionalizados portadores de esquizofrenia crônica
}

\author{
Comentado por: Beatriz Paiva Bueno de Almeida ${ }^{1}$, Maria Claudia Cunha ${ }^{2}$
}

Bowie CR, Harvey DP. Communication abnormalities predict functional in chronic schizophrenia: differential associations with social and adaptive functions. Schizophr Res. 2008;103(1-3):240-7.

Por mais de 200 anos, do século XII até meados do século $\mathrm{XX}$, a sociedade ocidental construiu e manteve instituições psiquiátricas, nas quais os sujeitos em sofrimento psíquico eram submetidos a longas internações e, com frequência, estavam sujeitos a procedimentos bastante invasivos. Tal situação acabou reiterando processos de abandono e de segregação social, embora tenha sido criada como suposta alternativa a eles ${ }^{(1)}$.

No Brasil, atualmente delineia-se um novo cenário na Saúde Mental. Este processo consiste na reorientação das instituições asilares que abrigam pacientes com transtornos mentais - dentre eles os portadores de esquizofrenia - visando o estabelecimento de uma ética de não-isolamento, consignada pela busca de alternativas para lidar com esses pacientes de maneira a promover a convivência e a circulação social dos mesmos.

Nesse cenário, emerge a importância da comunicação na manutenção da vida em sociedade para os sujeitos portadores de doenças mentais, particularmente daqueles institucionalizados.

Nessa direção, no artigo em análise, os autores - ambos pesquisadores de departamentos de psiquiatria (Mount Sinai School of Medicine e Emory University School of Medicine), afirmam que as dificuldades de comunicação há muito tem sido reconhecidas como um dos sintomas da esquizofrenia, contudo, elas assumem diversas formas, que poderiam ser associadas a duas categorias de transtornos de pensamento, denominados como positivo e negativo. O primeiro é caracterizado por discurso desorganizado, no qual ocorre a redução do conteúdo expresso em palavras ou frases, respostas inadequadas a perguntas e desconexão com a realidade. No segundo ocorre a redução da produção verbal.

Os autores prosseguem, sugerindo que tanto o discurso desorganizado quanto a redução da produção verbal parecem ser traços estáveis na esquizofrenia, embora pacientes mais velhos, e com curso crônico e contínuo da doença, apresentem essas deficiências com maior gravidade em relação aos mais jovens. Contudo, acrescentam que apesar da constatação da

(1) Programa de Estudos Pós-graduados em Fonoaudiologia, Pontifícia Universidade Católica de São Paulo - PUC-SP, São Paulo (SP), Brasil.

(2) Faculdade de Ciências Humanas e da Saúde, Pontifícia Universidade Católica de São Paulo - PUC-SP, São Paulo (SP), Brasil.

Endereço para correspondência: Beatriz Paiva Bueno de Almeida. PUC - Programa de Pós Graduação em Fonoaudiologia. R. Ministro de Godoy, 969, $4^{\circ}$ andar, São Paulo (SP), Brasil, CEP: 05015-000. E-mail: lebealmeida@hotmail.com cronicidade do distúrbio de comunicação e da vasta literatura dedicada aos fatores etiológicos envolvidos nessa condição, suas implicações funcionais são pouco conhecidas nessa população de pacientes.

Partindo da hipótese de que existem relações entre esses dois subtipos de distúrbios de comunicação com as deficiências no desempenho das funções sociais e as funções instrumentais adaptativas - características típicas de sujeitos esquizofrênicos cronicamente institucionalizados - estabelecem o objetivo do estudo, a saber: caracterizar e analisar essas relações.

Para tal, analisaram uma amostra de 317 pacientes nessas condições (clínica e institucional) por dois anos e meio. Todos os sujeitos residiam em uma unidade de internação psiquiátrica pública e possuíam esquizofrenia ou transtorno esquizoafetivo, segundo o DSM-IV. Foram selecionados somente os pacientes com pelo menos seis anos de escolaridade, de maneira a excluir aqueles que poderiam ter algum atraso significativo na aprendizagem, e os mesmos foram submetidos ao Mini Exame de Estado Mental (MEM).

Acrescenta-se que o $\mathrm{MEM}^{(2)}$ é um importante instrumento de rastreio de comprometimento cognitivo, pois clinicamente pode ser utilizado na detecção de perdas nesse nível, no seguimento evolutivo de doenças mentais e no monitoramento de respostas ao tratamento ministrado. Como instrumento de pesquisa ele tem sido largamente empregado em estudos epidemiológicos populacionais, integrando várias baterias de avaliação neuropsicológica.

Adotados esses procedimentos, a casuística da pesquisa ficou assim caracterizada: 198 do gênero masculino; 119 do gênero feminino; média de idade de 67,7 anos; escolaridade média de 11,5 anos; média de idade na primeira hospitalização aos 26,5 anos; todos medicados com pelo menos uma droga antipsicótica; intervalo médio de acompanhamento de 753 dias; $80 \%$ caucasianos; $16 \%$ afro americanos; $3 \%$ latinos americanos e menos de $1 \%$ asiáticos; todos falavam inglês, não possuíam déficits sensoriais e apresentavam comprometimento cognitivo de médio a moderado.

O transtorno de comunicação foi avaliado com a escala de avaliação de pensamento, linguagem e comunicação (TCL), a partir da coleta de dados no período de cinco a dez minutos de conversa com o pesquisador, bem como por meio da observação da comunicação durante as outras situações de avaliação clínica. Dentre os 18 subtipos de transtornos de linguagem elencados no TCL, o discurso desorganizado e a redução da produção verbal foram selecionados para análise. 
A incapacidade funcional foi avaliada com o teste de Avaliação do Funcionamento Social e Adaptativo (SAFE) que consiste em uma escala de 17 itens projetada para uso em contexto de internação, e descreve os desempenhos quanto a: 1. relacionamento social e interpessoal, 2. auto cuidado instrumental e 3. recursos de controle da impulsividade; não sendo este último avaliado no estudo.

Explicitando os conteúdos de cada subitem, quanto às relações sociais e interpessoais, seis comportamentos sociais foram considerados: comunicação (falar ao telefone e escrever cartas), dialogia (condutas verbais e não verbais), habilidades sociais (solicitar ajuda ou localização), educação social (cortesia), participação social (interação com pares) e vínculos de amizade. Quanto ao autocuidado: vestir-se, comer e participar de atividades recreativas.

Os resultados apontaram que a redução da produção verbal e o discurso desorganizado estabeleceram relações diferenciais com comportamentos sociais e adaptativos específicos. A análise estatística revelou associações significativamente elevadas entre discurso desorganizado e descortesia social, e entre redução da produção verbal e isolamento social, com ênfase em dificuldades em estabelecer vínculos de amizade. Ambos os subtipos de transtornos de comunicação também estabeleceram correlações significativas com os itens dialogia, comunicação e habilidades sociais.
Em síntese: os resultados do estudo em análise indicaram que, nessa população, os dois distúrbios da comunicação especificamente estudados foram persistentes e funcionalmente incapacitantes, na medida em que prejudicaram a sociabilidade desses pacientes.

Diante dessa constatação, salienta-se que o fonoaudiólogo pode contribuir de maneira significativa no atendimento clínico e na formulação de programas de intervenção dirigidos a esses pacientes, dadas as especificidades teóricas e técnicas da área. Em contrapartida, observa-se que no Brasil poucos estudos em Fonoaudiologia abordam o trabalho com pacientes esquizofrênicos nessa perspectiva.

Sendo assim, sugere-se o investimento em pesquisas fonoaudiológicas sobre o tema, as quais possam sustentar cientificamente as intervenções dirigidas a esses pacientes, estejam eles institucionalizados ou não.

\section{REFERÊNCIAS}

1. Amarante, Paulo. Saúde mental e atenção psicossocial. $2 a$ ed. Rio de Janeiro: Fiocruz; 2008.

2. Brucki SM, Nitrini R, Caramelli P, Bertolucci PH, Okamoto IH. Sugestões para o uso do mini-exame do estado mental no Brasil. Arq Neuro-Psiquiatr. 2003;61(3B):777-81. 